\title{
openheart Subclinical atherosclerosis and silent myocardial ischaemia in patients with type 2 diabetes: a protocol of a clinico-observational study
}

\author{
D S Prasad, ${ }^{1}$ Zubair Kabir, ${ }^{2}$ K Revathi Devi, ${ }^{3}$ A K Dash, ${ }^{4}$ B C Das ${ }^{5}$
}

To cite: Prasad DS, Kabir Z, Devi KR, et al. Subclinical atherosclerosis and silent myocardial ischaemia in patients with type 2 diabetes: a protocol of a clinicoobservational study. Open Heart 2014;1:e000100. doi:10.1136/openhrt-2014000100

Received 27 February 2014 Revised 15 May 2014 Accepted 24 May 2014

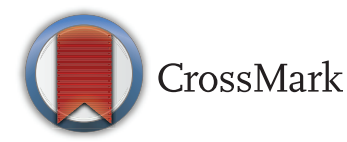

${ }^{1}$ Sudhir Heart Centre, Berhampur, Odisha, India ${ }^{2}$ Department of Epidemiology \& Public Health, University College Cork, Cork, Ireland ${ }^{3}$ Diabetes Clinic, Sudhir Heart Centre, Berhampur, Odisha, India

${ }^{4}$ Department of Pathology, MKCG Medical College, Berhampur, Odisha, India ${ }^{5}$ Department of Community Medicine, Kalinga Institute of Medical Sciences,

Bhubaneshwar, Odisha, India

Correspondence to

Dr D S Prasad;

sudhir_heartcare@hotmail. com

\section{ABSTRACT}

Introduction: Atherosclerotic cardiovascular disease is a significant modifiable complication in patients with diabetes and subclinical atherosclerosis is considered a surrogate marker of future vascular events. The clustering of cardiometabolic-risk factors in patients with diabetes and cardiovascular disease is increasingly being recognised. Recent evidence indicates that $20-50 \%$ of asymptomatic patients with diabetes may have silent coronary heart disease. However, the identification of subclinical atherosclerosis and silent myocardial ischaemia in patients with diabetes has been less well-explored, especially in low-resource population settings where cost-effective non-invasive clinical tools are available. The objective of this study is to identify patients with physician-diagnosed diabetes who are at risk of developing future cardiovascular events measured as subclinical atherosclerosis and silent myocardial ischaemia in an urban population of Eastern India.

Methods and analysis: This is a cross-sectional clinico-observational study. A convenience sampling of approximately 350 consecutive patients with type 2 diabetes based on predefined inclusion and exclusion criteria will be identified at an urban diabetes center. This estimated sample size is based on an expected prevalence of silent myocardial ischaemia of $25 \%$ $( \pm 5 \%)$, we computed the required sample size using OpenEpi online software assuming an $\alpha$ level of 0.05 $(95 \% \mathrm{Cl})$ to be 289 . On factoring $20 \%$ non-response the estimated sample size is 350 . Previously validated questionnaire tools and well-defined clinical, anthropometric and biochemical measurements will be utilised for data collection. The two primary outcomes - subclinical atherosclerosis and silent myocardial ischaemia will be measured using carotid intima-media thickness and exercise tolerance testing, respectively. Descriptive and multivariate logistic regression statistical techniques will be employed to identify 'at risk' patients with diabetes, and adjusted for potential confounders.

Ethics and dissemination: Ethical approval was granted by the institutional review board of Kalinga Institute of Medical Sciences, Bhubaneshwar, India. Data will be presented at academic fora and published in peer-reviewed journals.

\section{KEY MESSAGES}

South Asians apparently have a unique cardiometabolic risk profile especially in the context of Diabetes disorder. However, there is limited information on the clinical implications of exploring such clustering of cardiometabolic risk profile among Diabetes patients in South Asian populations

- This study protocol examines two clinical but modifiable outcomes - Subclinical Atherosclerosis and Silent Myocardial Ischemia in asymptomatic Diabetes patients employing cost-effective noninvasive diagnostic tools - carotid intima-media thickness and exercise tolerance testing measurements, respectively.

- The study findings will provide additional insights into expanding the existing knowledge base for policy planners to consider implementing cost effective guidelines in resource scarce health care settings for identifying 'at risk' diabetic patients.

\section{INTRODUCTION}

Diabetes rates are rapidly increasing in South-East Asia, with India and rest of South-East Asia bearing the major burden, 65 and 72 million, respectively, accounting for about one-fifth of all cases worldwide. ${ }^{1}$ Recent urban population studies from India showed a relatively high prevalence of diabetes ${ }^{2-4}$ and other cardiometabolic risk factors such as hypertension, central obesity and physical inactivity across all socioeconomic groups. ${ }^{5} \quad 6$ Earlier studies-the Multiple Risk Factor Intervention Trial (MRFIT) and the United Kingdom Prospective Diabetes Study (UKPDS) - have clearly established the underlying link between cardiometabolic risk factors and the increased risk of cardiovascular events in patients with diabetes. Recent evidence suggests that targeting interventions at the clustering of cardiovascular risk factors is more effective in preventing macrovascular disease rather than 
treatment of hyperglycaemia alone. ${ }^{7}$ Moreover, an increment in the prevalence of diabetes indirectly implicates an escalating coronary risk, particularly when diabetes and coronary heart disease (CHD) share common risk factors, ${ }^{8} 9$ thus confirming earlier evidence, ${ }^{10}{ }^{11}$ and also consistent with most recent evidence. ${ }^{12} 13$ Therefore, it is important to identify cardiometabolic risk factors within patients with diabetes who may be potentially prone to future cardiovascular events.

Furthermore, patients with diabetes have 2-4-fold increased risk of macrovascular disease compared with patients without diabetes, with CHD and stroke being the most common causes of death in patients with diabetes. ${ }^{14}$ CHD has been observed to affect two to three decades earlier in patients with diabetes as opposed to individuals without diabetes. Progression of coronary and peripheral vascular atherosclerosis is one of the most frequent and chronic complications of diabetes mellitus. ${ }^{15}$ Nevertheless, atherosclerotic cardiovascular disease (CVD) is a significant modifiable complication of altered glucose metabolism that also leads to considerable disease burden, increased health services use and premature morbidity and mortality. ${ }^{16}{ }^{17}$ However, patients with diabetes are often asymptomatic with myocardial ischaemia which is commonly seen in a later stage when it becomes clinically manifest. ${ }^{18}$

The recent evidence indicates that $20-50 \%$ of patients with asymptomatic diabetes have silent $\mathrm{CHD} .{ }^{19}$ Once CHD is symptomatic in diabetes, morbidity and mortality are high and significantly worse than in patients without diabetes. ${ }^{18}$ Hence, the advancement made in detection and treatment of CHD allows reconsidering the noninvasive and cost-effective screening of subclinical atherosclerosis by quantitative measurements of carotid intimamedia thickness (CIMT) ${ }^{20}{ }^{21}$ and silent myocardial ischaemia by exercise stress testing ${ }^{18}{ }^{22}$ for early cardiovascular risk analysis, thus facilitating an effective therapeutic intervention in patients with high-risk diabetes. Consequently, such early warning signs may potentially lead to a decline in CVD-related disability and death. Therefore, it is vital to detect subclinical atherosclerosis early to prevent future vascular events in patients with diabetes, considering that CVD and diabetes share common cardiometabolic risk factors. We aim to conduct a risk-assessment study in an urban population in Eastern India among patients with diabetes identified through clinical assessment over a period of 3 months, with the following two main study objectives:

1. To determine prevalence and to identify risk factors for silent myocardial ischaemia in patients with asymptomatic diabetes.

2. To assess if CIMT is a surrogate marker of future vascular events in patients with diabetes.

\section{METHODS AND ANALYSIS}

\section{Study design}

A cross-sectional clinico-observational study.

\section{Study setting}

Berhampur Municipal Corporation, an important city of Odisha state one of the underdeveloped regions of Eastern India bordering an affluent state of Andhra Pradesh of Southern India. Therefore, the residents here are a diverse mix of socioeconomic class, language, faith and customs.

\section{Study population}

The study will be a clinic-based observational study on 350 consecutive patients with type 2 diabetes (Diabetes) attending the diabetic clinic at Sudhir Heart Centre, Berhampur, India, over a period of 3 months.

\section{Inclusion/exclusion criteria}

Inclusion criteria:

A. Patients with diabetes (based on WHO criteria).

B. Patients with diabetes on treatment (oral antidiabetic agents or insulins).

C. Normal resting ECG.

Exclusion criteria:

A. Patients with diabetes and cerebrovascular events.

B. Patients with diabetes and cardiovascular events such as CHD and congestive cardiac failure.

C. Patients with diabetes and chronic kidney disease.

\section{Primary outcome measures}

Silent myocardial ischaemia (based on exercise tolerance testing) and subclinical atherosclerosis (identified through carotid ultrasound based quantitative intimamedia measurements of the carotid artery).

\section{Estimated sample size}

Current estimates of silent myocardial ischaemia in patients with diabetes among South Asian populations are less well-documented. Based on literature review, ${ }^{23}$ we assumed a prevalence of silent myocardial ischaemia among patients with diabetes to be $25 \%$. Based on this prevalence $( \pm 5 \%)$, we computed the required sample size using OpenEpi online software assuming an $\alpha$ level of 0.05 (95\% CI) to be 289. On factoring $20 \%$ nonresponse the estimated sample size is $350 .{ }^{24}$ The present study will be a sequel to our previous community-based study for knowing the prevalence of diabetes and impaired glucose tolerance (IGT) in the same locale. ${ }^{25}$ We will take adequate care to ensure good response rate by explaining the recruited participants about the importance of study to self and also to the community at large.

\section{Data collection}

Previously validated questionnaire tool that was applied to earlier studies will be used. ${ }^{5}$ In short, we will employ the WHO stepwise approach for data collection. ${ }^{25}$ Demographic, socioeconomic and self-reported behavioural information (smoking, alcohol, physical activity and diet), objective measures of anthropometry (height, weight, waist and hip circumferences), biochemical 
(plasma glucose, glycosylated haemoglobin, total cholesterol, triglycerides, high-density lipoprotein (HDL) cholesterol (HDLC), uric acid and creatinine levels) and ECG readings will also be obtained. Health conditions will be documented based on self-reported history of diabetes, hypertension and CVD (chest pain, heart attack or stroke). Family history for all of the above conditions will be collected.

\section{Blood pressure}

Blood pressure will be recorded in the sitting position in both arms to the nearest $2 \mathrm{~mm} \mathrm{Hg}$ using a mercury sphygmomanometer. Two readings will be taken $5 \mathrm{~min}$ apart, and the mean of two will be taken as the blood pressure.

\section{Anthropometric measurements}

Height will be measured with a tape to the nearest centimetre. All the participants will be expected to stand vertical with no shoes with their back against the wall, heels together and eyes focused forward.

Weight will be considered with a traditional spring balance that is kept on a concrete flat surface. The scale is checked every day and calibration will be carried out with 'known' weights. Participants will be asked to wear minimal clothing and weight will be recorded to the nearest $0.5 \mathrm{~kg}$.

Body mass index (BMI) will be considered using the formula: observed weight divided by height squared $\left(\mathrm{kg} / \mathrm{m}^{2}\right)$.

Waist circumference will be measured using a nonstretchable measuring tape. All the participants will be asked to stand upright in a comfortable pose with both feet together on a horizontal surface. Waist girth will be noted as the lowest horizontal girth between the costal margins and the iliac crests at least respiration.

Hip circumference will be noted as the maximum circumference at the level of the greater trochanter (the widest portion of the hip) on both sides. Measurements will be noted to the nearest centimetre.

Waist and hip ratio will be obtained by dividing the waist circumference $(\mathrm{cm})$ by the hip circumference $(\mathrm{cm})$.

\section{ECG}

The suggested procedure for obtaining a resting ECG, and the technical requirements for a suitable ECG are elaborated in the reference manual for the Minnesota code, and the same will be followed for all the participants. ${ }^{26}$ ECG changes suggestive of ST segment depression (Minnesota 4-1 to 4-2) or $Q$ wave changes (Minnesota codes $1-1-1$ to $1-1-7$ ) or $\mathrm{T}$ wave changes (Minnesota codes 5-1 to 5-3) or (Minnesota code 7-1-1) for complete left bundle branch block will be noted. ${ }^{27}$

\section{Tread mill test}

All the patients with diabetes included in the study, will undergo an exercise tolerance testing under Bruce protocol and will be separated into two groups according to the positive or negative test results for provocable ischaemia. Planar or down-sloping ST segment depression $>1 \mathrm{~mm}$ is the generally dependable indicator of exercise-induced ischaemia. ${ }^{28}$

1. No CHD: exercise stress test negative.

2. CHD: this group of patients having a positive exercise tolerance test result will be labeled as having silent ischaemia.

\section{Carotid ultrasound}

Quantitative measurements of CIMT by carotid ultrasound using the method as advised by the American Society of Echocardiography. ${ }^{21}$ Intimal medial measurements of the carotid artery are acknowledged as a standard of atherosclerosis in many clinical and epidemiological studies and are extensively used. ${ }^{29}$ The standard higher limit of intimal medial thickness is put at $0.6 \mathrm{~mm}$ for these studies. An increment of about $0.1 \mathrm{~mm}$ or more is linked with an amplified risk of myocardial infarct and/or cerebrovascular disease incidence by about $2-6$ times. ${ }^{30}$

\section{Biochemical investigations}

Biochemical investigations will be conducted on a sample of blood collected from each participant with a minimum $10 \mathrm{~h}$ of an overnight fast. All biochemical parameters will be performed using enzymatic kits as per the following methods; plasma glucose by glucose oxidaseperoxidase method, ${ }^{31}$ glycosylated haemoglobin ${ }^{32}$ by ion exchange resin method, total cholesterol by cholesterol oxidase (CHOD)/phenol-aminophenazone (PAP) method, ${ }^{33}$ triglycerides by glycerol-3-phosphate oxidase/ PAP method ${ }^{34}$ and high density lipoprotein cholesterol (HDLC) by polyethylene glycol/CHOD-PAP method, ${ }^{35}$ low-density lipoprotein (LDL) and very LDL will be calculated using formulae of Freidewald et al. ${ }^{36}$ Non-HDLC will be calculated using the formula (total cholesterol -HDLC). In addition other parameters such as uric acid $^{38}$ by uricase/PAP method, urea ${ }^{39}$ by modified Berthelot method and creatinine ${ }^{40}$ by modified Jaffe's method will also be carried out.

\section{Definitions of study variables}

Diabetes mellitus

Definition and diagnosis of diabetes mellitus and intermediate hyperglycaemia was based on the Report of a WHO/International Diabetes Federation (IDF) Consultation, Geneva.

Diabetes will be considered as those diagnosed by a physician and are on oral hypoglycaemic agents/insulin and/or those who had a fasting plasma glucose level of $126 \mathrm{mg} / \mathrm{dL} \quad(\geq 7.0 \mathrm{mmol} / \mathrm{L})$ or $2 \mathrm{~h}$ plasma glucose $\geq 200 \mathrm{mg} / \mathrm{dL}(11.1 \mathrm{mmol} / \mathrm{L})$ and IGT as fasting plasma glucose of $<7.0 \mathrm{mmol} / \mathrm{L}(126 \mathrm{mg} / \mathrm{dL})$ and $2 \mathrm{~h}$ plasma glucose of $\geq 7.8$ and $<11.1 \mathrm{mmol} / \mathrm{L} \quad(140 \mathrm{mg} / \mathrm{dL}$ and $200 \mathrm{mg} / \mathrm{dL}){ }^{41}$ 


\section{Microalbuminuria}

An unusually augmented excretion rate of albumin in the urine in the range of $30-299 \mathrm{mg} / \mathrm{g}$ creatinine is considered as microalbuminuria. ${ }^{42}$ It is frequently encountered and known as a cardiovascular risk indicator in diabetic populations. Microalbuminuria is supposed to be due to underlying generalised endothelial dysfunction.

\section{Hypertension}

Hypertension: based on physician diagnosis and antihypertensive treatment or systolic blood pressure $\geq 140$ or diastolic blood pressure $\geq 90 \mathrm{~mm} \mathrm{Hg} .{ }^{43} 44$

\section{Dyslipidaemia}

Dyslipidaemia: based on the Third Report of the National Cholesterol Education Program (NCEP). ${ }^{45}$ Hypercholesterolaemia: serum cholesterol $>200 \mathrm{mg} / \mathrm{dL}$; hypertriglyceridaemia: serum triglycerides $>150 \mathrm{mg} / \mathrm{dL}$; low HDLC: males $\leq 40 \mathrm{mg} / \mathrm{dL}$ and females $\leq 50 \mathrm{mg} / \mathrm{dL}$.

\section{Metabolic syndrome}

Diverse criteria have been put forward by several organisations for defining metabolic syndrome. ${ }^{46}$ We have adopted the latest definition from a Joint Interim Statement of international organisations such as International Diabetes Federation; National Heart, Lung, and Blood Institute; American Heart Association; World Heart Federation; International Atherosclerosis Society; and International Association for the Study of Obesity ${ }^{47}$ and a Consensus Statement from Association of Physicians of India. ${ }^{48}$ Presence of any three of the following five cardiometabolic risk factors is essential for the diagnosis.

1. Waist circumference: males $\geq 90 \mathrm{~cm}$ and females $\geq 80 \mathrm{~cm}$.

2. Hypertriglyceridaemia $\geq 150 \mathrm{mg} / \mathrm{dL}(1.7 \mathrm{mmol} / \mathrm{L})$.

3. Low HDLC: males $<40 \mathrm{mg} / \mathrm{dL}(1 \mathrm{mmol} / \mathrm{L})$ and females $<50 \mathrm{mg} / \mathrm{dL}(1.3 \mathrm{mmol} / \mathrm{L})$.

4. Systolic blood pressure $\geq 130 \mathrm{~mm} \mathrm{Hg}$ and/or diastolic blood pressure $\geq 85 \mathrm{~mm} \mathrm{HG}$ or drug treatment for hypertension.

5. Fasting blood sugar $\geq 100 \mathrm{mg} / \mathrm{dL}(5.6 \mathrm{mmol} / \mathrm{L})$ or drug treatment for diabetes mellitus.

\section{Obesity/overweight}

Obesity and overweight: based on the revised criteria specific for Asian/Pacific populations. Value of BMI $\geq 23 \mathrm{~kg} / \mathrm{m}^{2}$ used to define overweight and $\geq 25 \mathrm{~kg} / \mathrm{m}^{2}$ to define obese. ${ }^{49} 50$

\section{Physical activity}

Data on physical activity will be collected with questions on job-related and leisure time activities and exercise such as mode of transport to place of work, nature of work, number of days and type of exercise per week. ${ }^{51}$

\section{Fruit/vegetable intake}

Consumption of fruit and vegetable will be ascertained. Intake of $<5$ servings a day will be considered as inadequate fruit intake. ${ }^{52}$

\section{Socioeconomic status}

Socioeconomic status of an individual will be based on educational status, occupation and per capita income. ${ }^{53}$

\section{Smoking}

Data on smoking status of individual participants will be collected to categorise them as current, former and never a smoker. ${ }^{5}$ Users of all types of tobacco products (combustible and non-combustible) who smoked every day or some days in the past 1 month will be considered a current smoker; those who have smoked less than 100 cigarettes/combustible products in their lifetime will be considered a non-smoker; a former smoker is one who did not smoke at the time of the interview and has stopped smoking more than a month ago.

\section{Analysis}

All statistical analysis will be carried out using SPSS software. All continuous variables (including CIMT) will be reported as means \pm SD. The prevalence rates of various cardiovascular risk factors in patients with diabetes will be given in percentages. The mean values and frequencies of various risk factors (variables) will be studied in the group as a whole and individually in the two subgroups, namely those with silent CHD and those without CHD employing Students t test (for continuous variables) and Pearson's chi-squared test $\left(\chi^{2}\right)$ for categorical variables. Significant predictors of silent CHD will also be estimated applying backward elimination of multivariate logistic regression. Next, within the patients with silent CHD, two risk groups (namely, high and low) based on known cardiometabolic risk factors will be compared and contrasted employing multivariate logistic regression analyses. Finally, mean difference and 1-SD difference in CIMT readings will be estimated to predict subclinical atherosclerosis in patients with diabetes employing multivariate logistic regression techniques simultaneously adjusting for potential confounders. Statistically significance will be assumed at $\mathrm{p}<0.05$.

\section{Clinical and public health implications of the study}

This proposed study has important clinical and public health implications considering that diabetes is fast emerging as an epidemic in low-income and middle-income nations. First, a baseline risk pattern of patients with diabetes who may be at risk of developing macrovascular events in the future can be identified and documented. Second, the unique nature of the study population is an important piece of information, thus complementing the existing body of evidence. Third, the study findings will be a better clinical management tool for identifying 'at risk' patients. Fourth, the study findings can be reproducible in different population 
settings for consistency. Future research must include a comprehensive analysis of patients with diabetes who may be at risk of developing vascular events in the future through longitudinal follow-up studies.

\section{Strengths and limitations of the study}

The main limitation of the present study is its crosssectional nature, thus precluding us from inferring a causal relationship. Residual confounding because of unknown and unmeasured confounder will be a methodological issue in this study. Other study limitations such as recall and measurement biases will be minimised through the administration of previously validated questionnaire tools, trained interviewers and with the help of calibrated diagnostic and anthropometric tools based on well-defined measurement criteria. However, the main strength of this study is the uniqueness of the study population and the diverse data collection ranging from sociodemographic, dietary, anthropometric to biochemical and clinical measurements.

\section{Ethics and dissemination}

The study will be in agreement with the Indian Council of Medical Research guidelines on bioethics. ${ }^{54}$ The study proposal was approved by the institutional review board of Kalinga Institute of Medical Sciences, Bhubaneshwar, India.

A written informed consent will be obtained from all participants prior to the study. All observations will be presented at regional, national and global fora and published in peer-reviewed journals. All upcoming publications will pursue the Strengthening the Reporting of Observational Studies in Epidemiology (STROBE) criteria. ${ }^{55}$ Patients and policymakers will be informed of results through presentations and reports (technical and non-technical). It is our hope that these findings will inform clinical and public health service development in a resource scarce healthcare setting.

Contributors DSP and KRD conceived and designed the study and wrote the protocol. ZK refined the study design and revised the protocol. AKD and BCD reviewed the literature and finalised the protocol. All the authors read and approved the final version of the protocol.

\section{Competing interests None.}

Patient consent Obtained.

Ethics approval Institutional review board of Kalinga Institute of Medical Sciences, Bhubaneshwar, India.

Provenance and peer review Not commissioned; externally peer reviewed.

Data sharing statement No additional data are available.

Open Access This is an Open Access article distributed in accordance with the Creative Commons Attribution Non Commercial (CC BY-NC 3.0) license, which permits others to distribute, remix, adapt, build upon this work noncommercially, and license their derivative works on different terms, provided the original work is properly cited and the use is non-commercial. See: http:// creativecommons.org/licenses/by-nc/3.0/

\section{REFERENCES}

1. IDF. Diabetes atlas. 6th edn. Brussels: International Diabetes Federation, 2013.

2. Prasad DS, Kabir Z, Dash AK, et al. Prevalence and risk factors for diabetes and impaired glucose tolerance in Asian Indians: a community survey from urban Eastern India. Diabetes Metab Syndr 2012;6:96-101.

3. Mohan V. Why are Indians more prone to Diabetes? J Assoc Physicians India 2004;52:468-74.

4. Hoskote SS, Joshi SR. Are Indians destined to be Diabetic? J Assoc Physicians India 2008;56:225-6.

5. Prasad DS, Kabir Z, Dash AK, et al. Coronary risk factors in South Asians: a prevalence study in an urban populace of Eastern India. CVD Prev Control 2010;5:125-32.

6. Gupta M, Brister S. Is South Asian ethnicity an independent cardiovascular risk factor? Can J Cardiol 2006;22:193-7.

7. Yki-Jarvinen $\mathrm{H}$. Management of type 2 diabetes mellitus and cardiovascular risk: lessons from intervention trials. Drugs 2000;60:975-83.

8. Kannel WB. Metabolic risk factors for coronary heart disease in women: perspective from the Framingham Study. Am Heart $J$ 1987;114:413-19.

9. Haffner SM, Lehto S, Ronnemaa T, et al. Mortality from coronary heart disease in subjects with type 2 diabetes and in nondiabetic subjects with and without prior myocardial infarction. $N$ Engl J Med 1998;339:229-34.

10. Bradley RF, Bryfolge JW. Survival of diabetic patients after myocardial infarction. Am J Med 1956;20:207-16.

11. Jarret RJ. Type 2(non insulin dependant) diabetes mellitus and coronary heart disease: chicken, egg or neither? Diabetologia 1984;26:99-102.

12. Pradeepa R, Nazir A, Mohan V. Type 2 diabetes and cardiovascular diseases: do they share a common soil? The Asian Indian experience. Heart Asia 2012;4:69-76.

13. Pasterkamp G. Methods of accelerated atherosclerosis in diabetic patients. Heart 2013;99:743-9.

14. Viberti $\mathrm{G}$. The need for tighter control of cardiovascular risk factors in diabetic patients. J Hypertens Suppl 2003;21:S3-6.

15. Sukhija R, Dhanwal D, Gambhir DS, et al. Silent myocardial ischaemia in patients with type II diabetes mellitus and its relation with autonomic dysfunction. Indian Heart J 2000;52:540-6.

16. Eckel RH, Kahn R, Robertson RM, et al. Preventing cardiovascular disease and diabetes: a call to action from the American Diabetes Association and the American Heart Association. Circulation 2006;113:2943-6.

17. Ryden L, Mellbin L. Glucose perturbations and cardiovascular risk: challenges and opportunities. Diab Vasc Dis Res 2012;9:170-6.

18. Wackers FJT, Young LH, Inzucchi SE, et al. Detection of silent myocardial ischemia in asymptomatic diabetic subjects: the DIAD study. Diabetes Care 2004;27:1954-61.

19. Di Carli MF, Hachamovitch R. Should we screen for occult coronary artery disease among asymptomatic patients with diabetes? J Am Coll Cardiol 2005;45:50-3.

20. O' Leary DH, Bots ML. Imaging of atherosclerosis: carotid intima-media thickness. Eur Heart J 2010;31:1682-9.

21. Stein JH, Korcarz CE, Hurst RT, et al. Use of carotid ultrasound to identify subclinical vascular disease and evaluate cardiovascular disease risk: a Consensus Statement from the American Society of Echocardiography Carotid Intima-Media Thickness Task Force. Endorsed by the Society for Vascular Medicine. J Am Soc Echocardiogr 2008;21:93-111.

22. Barthelemy O, Le Feuvre C, Timsit J. Silent myocardial ischemia screening in patients with diabetes mellitus. Arq Bras Endocrinol Metab 2007;51:285-93.

23. Valensi P, Lorgis L, Cottin Y. Prevalence, incidence, predictive factors and prognosis of silent myocardial infarction: a review of the literature. Arch Cardiovasc Dis 2011;104:178-88.

24. Lemeshow S, Hosmer DW, Klar J, et al. Adequacy of sample size in health studies. John Wiley and Sons, 1990.

25. Bonita R, De Courten M, Dwyer T, et al. Surveillance of risk factors for non communicable disease: the WHO STEPwise approach. Summary. Geneva: World Health Organisation, 2001.

26. Prineas RJ, Crow RS, Blackburn H. The Minnesota code manual of electrocardiographic findings: standards and procedures for measurement and classification. Littleton, MA: Wright-PSG, 1982.

27. Macfarlane PW. Minnesota coding and the prevalence of ECG abnormalities. Heart 2000;84:582-84.

28. Jonathan $\mathrm{H}$, Adam T. Exercise tolerance testing. $B M J$ 2002;324:1084-7. 
29. Sidhu PS, Desai SR. A simple and reproducible method for assessing intimal-medial thickness of the common carotid artery. $\mathrm{Br}$ J Radiol 1997;70:85-9.

30. Lees RS. Non-invasive detection of vascular function and dysfunction. Curr Opin Lipidol 1993;4:325-9.

31. Trinder $P$. Determination of glucose in blood using glucose oxidase as an alternative oxygen receptor. Ann Clin Biochem 1969;6:24-30.

32. Nathan DM, Singer DE, Hurxthal $\mathrm{K}$, et al. The clinical information value of the glycosylated hemoglobin assay. $N$ Engl $\mathrm{J} \mathrm{Med}$ 1984;310:341-6.

33. Allain CC, Poon LS, Chan CS, et al. Enzymatic determination of total serum cholesterol. Clin Chem 1974;20:470.

34. Fossati P, Principe L. Serum triglycerides determined colorimetrically with an enzyme that produces hydrogen peroxide. Clin Chem 1982;28:2077.

35. Donald WA, Jaysmith S. Six methods for isolating high density lipoprotein compared with the use of the reference for quantifying cholesterol in serum. Clin Chem 1985;31:746.

36. Freidewald WT, Levy RJ, Fredrickson . Estimation of the concentration of LDL Cholesterol in plasma without use of the preparative ultracentrifuge. Clin Chem 1972;18:499.

37. Wilson DE, Spiger MJ. A dual precipitation method for quantitative plasma lipoprotein measurement without ultracentrifugation. $J$ Lab Clin Med 1973;82:473.

38. Fossati P, Prencipe L, Berti G. Use of 3,5-dichloro-2-hydroxybenzenesulfonic acid/4-aminophenazone chromogenic system in direct enzymic assay of uric acid in serum and urine. Clin Chem 1980;26:227-31.

39. Fawcett JK, Scott JE. A rapid and precise method for the determination of urea. J Clin Pathol 1960;13:156-9.

40. Bowers LD, Wong ET. Kinetic serum creatinine assays. II. A critical evaluation and review. Clin Chem 1980;26:555-61.

41. Definition and diagnosis of diabetes mellitus and intermediate hyperglycemia: report of a WHO/IDF Consultation. Geneva: World Health Organization, 2006.

42. Toto RD. Microalbuminuria: definition, detection, and clinical significance. J Clin Hypertens (Greenwich) 2004;6(11 Suppl 3):2-7.

43. Chobanian AV, Bakris GL, Black HR, et al. Seventh report of the Joint National Committee on Prevention, Detection, Evaluation, and Treatment of High Blood Pressure. Hypertension 2003;42:1206-52.
44. Prasad DS, Kabir Z, Dash AK, et al. Prevalence and predictors of adult hypertension in an urban eastern Indian population. Heart Asia 2012;4:49-52.

45. Third Report of the National Cholesterol Education Program (NCEP). Expert Panel on Detection, Evaluation, and Treatment of High Blood Cholesterol in Adults (Adult Treatment Panel III) Final Report. Circulation 2002;106:3143-421.

46. Prasad DS, Kabir Z, Dash AK, et al. Prevalence and risk factors for metabolic syndrome in Asian Indians: a community study from urban eastern India. J Cardiovasc Dis Res 2012;3:204-11.

47. Alberti KGMM, Eckel RH, Grundy SM, et al. Harmonizing the metabolic syndrome. Circulation 2009;120:1640-5.

48. Misra A, Chowbey P, Makkar BM, et al. Consensus Statement for Diagnosis of obesity, abdominal obesity and the metabolic syndrome for Asian Indians and recommendations for physical activity, medical and surgical management. J Assoc Physicians India 2009;57: $163-70$.

49. The AsiaPacific Perspective. Redefining obesity and its treatment. Melbourne: World Health Organization. International Association for the Study of Obesity and International Obesity Task Force. International Diabetes Institute World Health Organization, Western Pacific Region, 2000.

50. Prasad DS, Kabir Z, Dash AK, et al. Effect of obesity on cardiometabolic risk factors in Asian Indians. J Cardiovasc Dis Res 2013;4:116-22.

51. Prasad DS, Das BC. Physical inactivity: a cardiovascular risk factor. Indian J Med Sci 2009;63:33-42.

52. $\mathrm{Ng} \mathrm{N}$, Minh HV, Juvekar S, et al. Using the INDEPTH HDSS to build capacity for chronic non-communicable disease risk factor surveillance in low and middle income countries. Glob Health Action 2009:2:10

53. Kumar N, Shekhar C, Kumar P, et al. Kuppuswamy's socioeconomic status scale-updating for 2007. Indian J Pediatr 2007;74:1131-2.

54. Ethical Guidelines for Biomedical Research on Human subjects. 3rd edn. New Delhi: The Indian Council of Medical research, 2006.

55. Vandenbroucke JP, Elm Ev, Altman DG, et al. Strengthening the reporting of observational studies in epidemiology (STROBE): explanation and elaboration. Ann Intern Med 2007;147:W163-94. 